$>$ Cette contribution porte sur les implications médicales et sociales du cancer chez le sujet âgé avec pour référence les études les plus récentes dans la population américaine. Cette présentation fait le point sur les effets positifs de la précocité du diagnostic et la prise en charge des sujets par une équipe de soignants, sur la problématique de la mise en œuvre des décisions et des choix thérapeutiques. La conclusion cible l'importance de l'âge fonctionnel du patient et la présence de «l'aidant naturel », sur lequel les pouvoirs publics devraient statuer. <

\section{Implications \\ médicales \\ et sociales \\ du cancer \\ chez le sujet âgé}

Lodovico Balducci

\section{On tue les vieux}

Cette présentation commencera par ce titre très provocateur d'un livre écrit ici à Marseille par mon ami Jacques Soubeyrand [1]. Mieux que ne le feraient les données scientifiques, ce livre a su exprimer une réalité moderne, un paradoxe de notre médecine. Celle-ci a su prolonger la vie, mais elle a aussi fait de nous des Étrangers à nous-mêmes et aux autres, selon le mot d'Albert Camus [2].

\section{Comment tue-t-on les vieux?}

La réduction de l'espace vital, les mégapoles, ne sont pas favorables à la personne âgée. Avec la disparition des quartiers, le développement des grands centres commerciaux, les vieux sont de plus en plus isolés.

Les traitements trop nombreux, superflus parfois, et à des doses excessives, sont cause fréquente de mortalité. Les hospitalisations prolongées entraînent une perte d'autonomie, et finalement accélèrent la mortalité des vieux.

À l'inverse, parce qu'on pensait que les sujets âgés ne pouvaient pas tolérer la chimiothérapie à la même dose que les sujets plus jeunes, on a omis de faire des traitements efficaces, et de ce fait on contribue aussi à «tuer les vieux » : les traitements anticancéreux insuffisants sont une des principales causes de mortalité des personnes âgées.

Le fait qu'ils aient été exclus des protocoles d'essais cliniques pose un grave problème qui a été mentionné

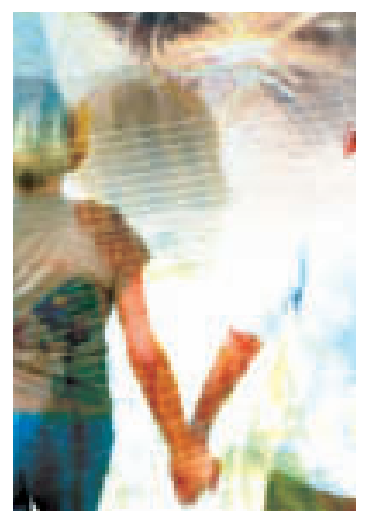

à de multiples reprises. Aujourd'hui, aux ÉtatsUnis, il n'existe plus de limite d'âge pour les essais cliniques, mais en pratique, on constate que les vieux sont toujours absents des études entreprises sur de nouvel-

Professeur de médecine et d'oncologie à l'Université de Floride, College of Medicine, en charge du programme d'oncogériatrie au H. Lee Moffitt Cancer Center Research Institute, Tampa, Floride. Department of Interdisciplinary Oncology, University of South Florida College of Medicine, Tampa, Floride. Directeur, Division of Geriatric Oncology Senior Adult Oncology Program, H. Lee Moffitt Cancer Center and Research Institute, 12902 Magnolia Dr MCC SA Program, Tampa, FL 33612, États-Unis. balducci@moffitt.usf.edu les molécules. Il serait donc souhaitable d'instaurer des essais cliniques spécialement dédiés aux sujets âgés. Enfin, la conduite du traitement et le suivi doivent se faire en concertation avec les proches, car la solitude du vieillard, la lassitude des familles, son abandon constituent un des facteurs aggravant le pronostic, qui préoccupent le médecin traitant et le gériatre.

\title{
Cancer et vieillissement
}

Une importante étude - pour ne donner qu'un seul exemple - a été réalisée montrant que l'âge n'intervient pas dans le pronostic du lymphome; le facteur décisif de survie dépend essentiellement du traitement: la chimiothérapie doit être prescrite à dose suffisante.

En 2000, les sujets âgés représentaient $12 \%$ de la population des États-Unis et avaient $50 \%$ de tous les cancers. En 2030, les sujets âgés représenteront $20 \%$ de la population et auront $70 \%$ de tous les cancers. 
Pour une population aussi nombreuse, si l'on ne sait pas soigner les cancers chez les sujets âgés, comment peut-on prétendre maîtriser le traitement des cancers?

Bien que la mortalité due aux cancers ait diminué, cette diminution est beaucoup plus lente que celle de la mortalité par maladies cardiovasculaires. Et, depuis l'an 2000, le cancer est devenu la cause de décès la plus commune jusqu'à 85 ans. Si ces personnes n'avaient pas eu de cancer, elles auraient pu vivre plus longtemps.

N'oublions pas que notre durée de vie est inférieure de 50 ans à notre espérance de vie théorique. Celle-ci est estimée aux alentours de 120 ans. Dans les années 1960, un Russe affirmait qu'il avait 155 ans et disait qu'il avait connu Napoléon (le seul problème était qu'il n'avait pas de certificat de naissance!). Mais Jeanne Calment, qui a été bien étudiée au cours de ses dernières années, a réellement vécu jusqu'à l'âge de 125 ans.

\section{Traitement du cancer chez le sujet âgé}

La mortalité liée au cancer a été réduite grâce au diagnostic précoce, à des protocoles de traitement plus efficaces et moins toxiques, et à de meilleures conditions générales. Elle a diminué en outre sous l'effet de la réduction des décès par cancer du sein (meilleure prévention, meilleurs traitements), de la prolongation de la survie des patients atteints de lymphome folliculaire, et du doublement de la survie du cancer métastatique de l'intestin. Cette amélioration peut et doit s'appliquer à l'ensemble de la population, sujets âgés compris.

Traiter un cancer chez un sujet âgé est une entreprise d'équipe. On ne peut pas décider de prescrire une chimiothérapie si le patient est seul et isolé. Pour un malade trop faible, incapable d'éviter les chutes durant la nuit, de téléphoner ou de signaler toutes les complications éventuelles du traitement, une présence est indispensable. II est donc très important de trouver un environnement satisfaisant pour la période du traitement.

On doit aussi se demander quel est le but final du traitement chez le sujet âgé. La guérison est l'objectif principal. Mais dans certains cas, il est difficile de savoir s'il sera possible de l'obtenir.

Quant à la prolongation de la survie avec cancer, ceci est un autre problème. Nous ne l'appréhendons encore que de façon rudimentaire

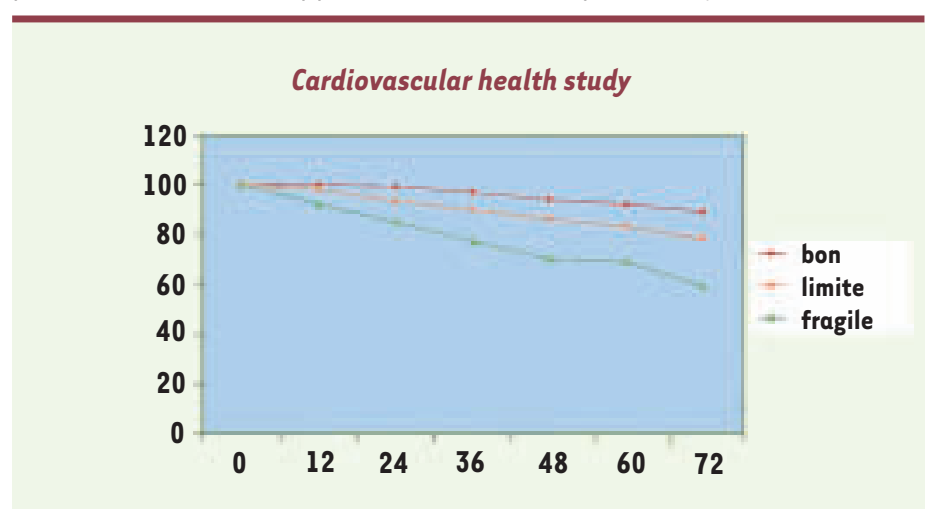

Figure 1. Survie comparée des sujets âgés de la cohorte de la CVH en fonction des critères de fragilité de Fried en trois groupes. Rouge: bonnes conditions physiologiques; orange : limites; vert : fragiles (tiré de [3]). et imparfaite. Il faut en effet s'interroger sur les conséquences générales de la prolongation de la survie.

Les études actuelles montrent que la prévalence des comorbidités et de l'invalidité ont augmenté. De plus, les complications précoces et tardives du traitement sont très graves, surtout les complications neurologiques.

Enfin, le coût économique, social et psychologique de l'organisation des services de soins et des services de gériatrie a augmenté. Les dépenses de soins dédiées aux personnes âgées sont nettement plus élevées en raison de l'assistance dont ils ont besoin.

\section{Comment organiser la prévention}

et le traitement du cancer chez le sujet âgé ?

La guérison, la prolongation de la survie doivent tendre vers une survie active.

Elle implique la diminution de la morbidité et la prise en charge des symptômes.

Qu'est-ce que la survie active?

En admettant que la survie atteigne 100 ans, il faut qu'autour de l'âge de 70 ans, $60 \%$ de la population ait peu d'invalidité et $40 \%$ seulement une morbidité. À la fin de sa vie, $100 \%$ de la population cumule invalidité et morbidité.

Certes, la mort est inévitable, mais dans l'idéal, il faudrait réduire morbidité et invalidité. Celles-ci devraient être limitées aux 30 dernières minutes plutôt qu'aux 30 dernières années de la vie !

\section{Décisions et choix thérapeutiques}

Pour aborder le problème du traitement du sujet âgé, il faut d'abord répondre aux questions suivantes:

- Le patient va-t-il mourir de son cancer ou avec son cancer?

- Le patient va-t-il souffrir des conséquences du cancer?

- Le patient est-il capable de tolérer le traitement de son cancer?

- Quelles seront les conséquences précoces et tardives du traitement?

Il faut donc avoir une connaissance de l'âge physiologique, et non de l'âge chronologique du patient.

Il faut aussi connaître le stade et l'agressivité du cancer, ainsi que l'espérance de vie et la réserve fonctionnelle du patient: on peut alors évaluer la probabilité d'une prolongation de la vie active.

Car le vieillissement est une perte d'entropie (capacité à produire de l'énergie). II est aussi une perte de fractalité. - La perte d'entropie signifie l'augmentation du risque de développer des maladies et la diminution de la tolérance au stress. 
- La fractalité est un concept plus difficile à expliciter. Que signifie ce mot qui ressemble à fatalité ? La fractalité est un processus dynamique qui se manifeste dans toutes les particules et ce, des plus élémentaires aux plus complexes. Des protons aux galaxies, la fractalité existe. L'arbre en est l'exemple le plus symbolique. On sait que le développement qu'il porte en lui va donner des branches et que chaque branche sera à l'origine d'une autre branche, mais on ne peut prévoir la longueur et ni le nombre des branches qui vont se former.

La fractalité est aussi une capacité humaine mais qui se perd avec le vieillissement. Ainsi ce cas présenté en Israël par une gériatre : un homme de 85 ans marchait très bien quand il était soutenu par une infirmière, mais il tombait quand il n'était plus soutenu: la perte de fractalité est associée à la dépendance fonctionnelle et aux syndromes gériatriques.

L'entropie et la fractalité ne sont pas mesurables chez l'homme. On ne peut qu'évaluer les conséquences de leur perte.

Ce type de vieillissement pathologique relève en partie de processus inflammatoires et immunitaires.

Ferrucci a démontré qu'avec le vieillissement, la concentration des cytokines pro-inflammatoires dans le sang augmente. Cohen quant à lui a prouvé qu'il existait une connexion entre l'inflammation chronique et le vieillissement.

J'ai photographié mes tantes jumelles pour leur $90^{\circ}$ anniversaire. Ces deux femmes possédant le même matériel génétique ont vécu dans le même village, ont exercé la même profession d'institutrice, mais à 90 ans, elles étaient très différentes. L'une était complètement démente, l'autre est restée autonome. Si j'avais eu à décider d'un traitement contre un cancer, j'aurais donné une chimiothérapie adjuvante à cette dernière, mais je ne crois pas que je l'aurais donnée à la première.

Ainsi, le bilan gériatrique inclut l'analyse de l'autonomie, des co-morbidités, des syndromes gériatriques et des ressources sociales.

L'autonomie comprend les activités quotidiennes: manger, faire sa toilette, s'habiller, aller à la salle de bains et se déplacer d'une pièce à l'autre, et être continent. Un patient incapable d'accomplir l'une ou plusieurs de ces activités, en état de déchéance physiologique ne sera pas un très bon candidat pour la chimiothérapie adjuvante. Tout au plus peut-on utiliser une chimiothérapie palliative, mais son espérance de vie sera de toute manière très réduite.

Le Docteur Lucia Fratino a été la première à démontrer que l'incapacité d'assurer l'une des activités suivantes: utiliser les moyens de transport, prendre ses médicaments, faire ses courses, faire la cuisine, gérer son argent, utiliser le téléphone, est associée à un risque augmenté de la toxicité de la chimiothérapie.

Les syndromes gériatriques (avec chutes répétées, incapacité à s'alimenter, négligence d'une hygiène élémentaire, incontinence, délire, démence) sont le signe d'un vieillissement très avancé.

Les co-morbidités les plus significatives sont les maladies cardiovasculaires, l'anémie, la dépression et le cancer. L'anémie chez le sujet âgé est associée à fréquence des chutes, et de la démence et à une augmentation de la mortalité. La dépendance fonctionnelle va de pair avec une augmentation de la toxicité de la radiothérapie. Car la plupart des traitements nécessite un nombre normal de globules rouges. Quand l'hémoglobine chute, la proportion de médicaments libres dans la circulation augmente.

Ces données sont essentielles pour estimer l'espérance de vie et prendre des décisions thérapeutiques.
Mitzinski propose un test pour calculer l'index de fragilité et l'âge chronologique. Il permet de mesurer l'âge fonctionnel des patients.

Une grande étude de cohorte américaine (cardiovascular health study) a suivi 85000 patients de 65 ans et plus pendant 11 ans. Les patients ont pu être divisés en 3 groupes selon 5 paramètres très simples. Les 3 groupes avaient une mortalité très différente après 6 années (Figure 1).

\section{Conclusions}

Le traitement des sujets âgés atteints de cancer dépend de leur âge fonctionnel, et aussi de la présence auprès d'eux de celui que j'appellerais «l'aidant naturel », la personne familière qui prend soin de lui.

II s'agit le plus souvent des épouses, qui ont souvent ellesmêmes des problèmes de santé, ou de filles, célibataires ou sans enfants. Quand cette fille est mère de famille, ce travail est une charge supplémentaire, ayant des répercussions sur la vie sociale et professionnelle, II s'agit parfois, mais plus rarement, d'un fils ou d'un(e) parent(e) proche.

II y aurait beaucoup à dire sur les droits, les aides que l'on devrait apporter à ces personnes irremplaçables que sont les «aidants naturels. » Car l'ensemble de la population sera immanquablement confronté à ce problème et devra, un jour ou l'autre, s'investir dans ce rôle. II faut donc que les médecins, les pouvoirs publics reconnaissent ce statut d'aidant naturel, exigent de lui un minimum de compétences, mais aussi l'impliquent dans les décisions à prendre, et lui facilitent, au maximum et par tous les moyens, l'accomplissement de cette tâche, surtout quand elle concerne des personnes atteintes de cancer. $\diamond$

\section{SUMMARY}

Medical and social involvements of the cancer in the elderly

This contribution concerns the medical and social involvements of the cancer in the elderly with for reference the most recent studies in the American population. The presentation reviews the positive effects of the precocity of the diagnosis and the coverage of the patients by a nursing team, the question of implementation about decisions and therapeutic choices. The conclusion targets the importance of the functional age of the patients and the presence of natural helping, on which the authorities should rule. $\diamond$

\section{RÉFÉRENCES}

1. Fernandez C, Pons T, Prédali D, Soubeyrand J. On tue les vieux. Paris : Fayard, 2006.

2. Camus A. L'Étranger. Paris : Gallimard, $1972: 186 \mathrm{p}$.

3. Fried LP, Tangen CM, Walston J, et al. Frailty in older adults: evidence for a phenotype.J Gerontol 2001 ; 56 : 146-57.

TIRÉS À PART

L. Balducci 